\title{
Gate Spacer Investigation for Improving the Speed of HF Carbon Nanotube-based Field-Effect
}

\section{Transistors}

Martin Hartmann ${ }^{* \pm 1,2}$, Jana Tittmann-Otto ${ }^{1,2}$, Simon Böttger ${ }^{1}$, Georg Heldt ${ }^{3}$, Martin Claus ${ }^{2}$, Stefan E. Schulz ${ }^{1,2,3}$, Michael Schröter ${ }^{4}$, Sascha Hermann ${ }^{1,2,3}$

${ }^{1}$ Chemnitz University of Technology, Center for Microtechnologies, 09126 Chemnitz

${ }^{2}$ Center for Advancing Electronics Dresden CFAED, 01062 Dresden

${ }^{3}$ Fraunhofer Institute for Electronic Nano Systems, Department Back-End of Line, 09126 Chemnitz

${ }^{4}$ Dresden University of Technology, Chair for Electron Devices and Integrated Circuits, 01062

Dresden

\section{Corresponding Author}

*E-mail: martin.hartmann@zfm.tu-chemnitz.de Tel: +49 37153132347 


\section{Transfer characteristics of HF CNTFETs with variating gate lengths}

Comparing the DC transfer characteristics (TC) of HF CNTFETs with different gate lengths and a constant channel length of $280 \mathrm{~nm}$ as well as a symmetrical gate position within the channel exhibits a rather similar trend for all devices. Five exemplary HF CNTFETs TCs are shown in figure S1. For all devices the off-current (at $2 \mathrm{~V}_{\mathrm{G}}$ ) is high giving a low on-off ratio. This is typical for dense CNT assemblies with CNT bundles and CNT crossings especially in combination with electrostatic doping effects e.g. by water and oxygen molecules. However, we want to investigate the potential of CNTFETs for analog applications with special focus on their speed in contrast to digital FETs where a high on-off current ratio is essential. In the main manuscript we have shown a reduction of device speed for a gate to source/ drain electrode underlap due to a reduction of gm. The heterojunction between the metal covered CNT segment and the uncovered CNT segment yields a Schottky-like barrier which is modified by the gate potential. A gate electrode underlap fosters the formation of

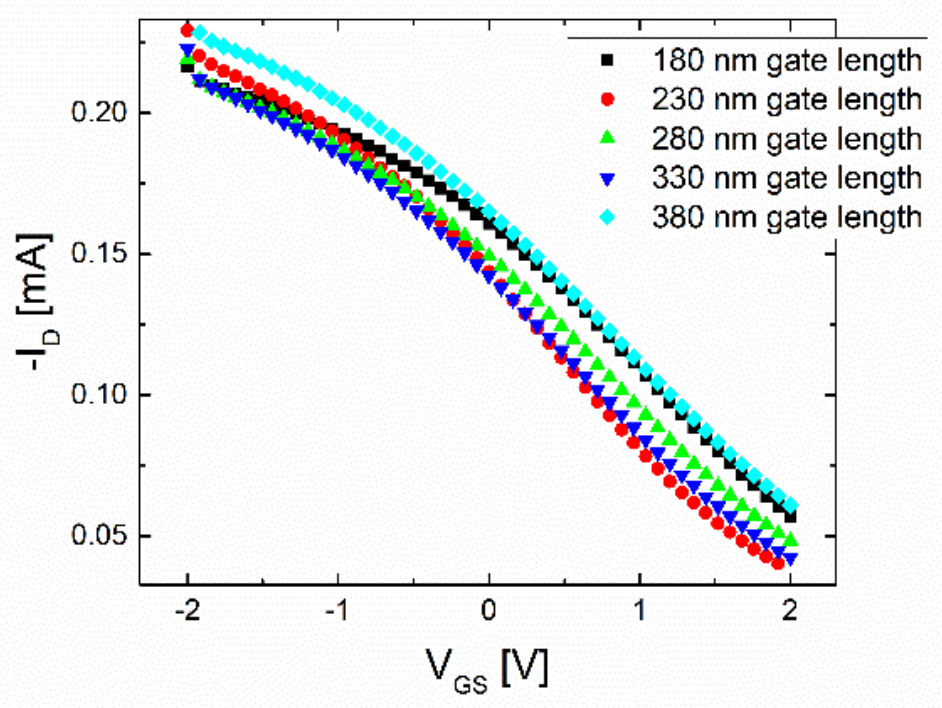

Figure S1: transfer characteristics of exemplary HF CNTFETs with a channel length of $280 \mathrm{~nm}$ and varying gate length, measured at a $D-S$ voltage of $-0.1 \mathrm{~V}$

near contact barriers and reduces the overall current. This can be partly seen in the TC presented in figure S1. Despite the TC of the HF CNTFET with $380 \mathrm{~nm}$ gate length the highest on-current (at $-2 V_{G}$ ) features the device with $230 \mathrm{~nm}$ gate length, which also shows the highest speed. For both increased as well as decreased gate lengths the on-current is decreasing. The DC gm follow the trend of the HF gm. However, the device with $380 \mathrm{~nm}$ gate length shows a current offset leading to an enhanced overall current. The exact phenomenon is not exactly clear yet and will be investigated in the future together with the impact of charge traps onto the device performance.

\section{Transfer characteristics of HF CNTFETs with varying source spacer}

The TC of the HF CNTFETs with varying gate positions within the channel and hence different Sspacer and a constant channel length of $280 \mathrm{~nm}$ and gate length of $230 \mathrm{~nm}$ feature again rather low on-off current ratios. Following the arguments above we see again the highest on-current at $0 \mathrm{~nm} \mathrm{~S}$ spacer yields the highest $\mathrm{f}_{\mathrm{T}}$. For both increased and decreased $\mathrm{S}$-spacer, gm as well as the on-current is decreasing. 


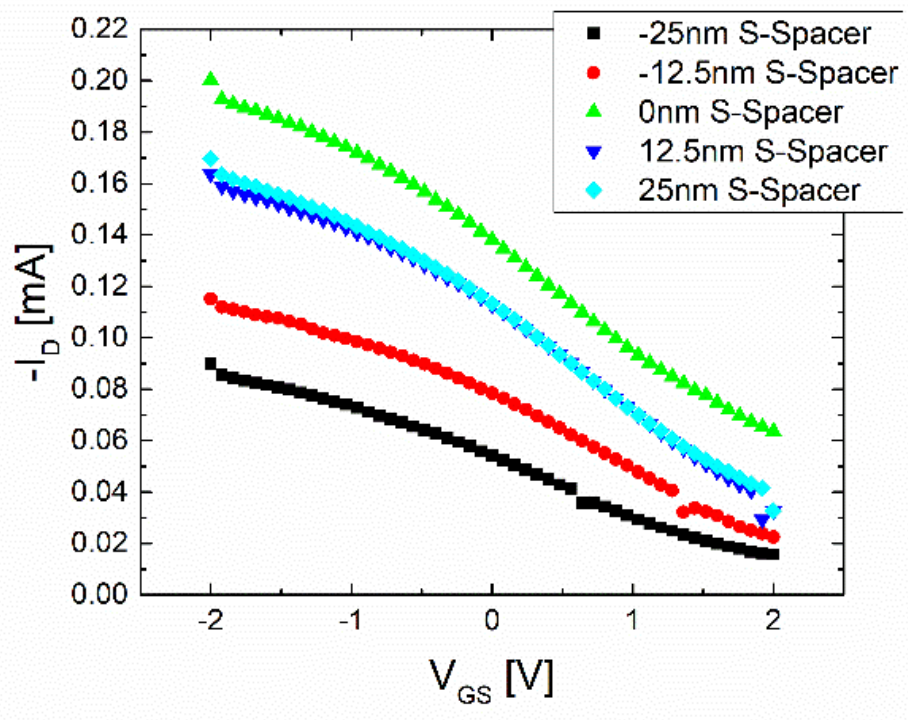

Figure S2: transfer characteristics of exemplary HF CNTFETs with a channel length of $280 \mathrm{~nm}$ and varying $S$-spacer, measured at a $D$-S voltage of $-0.1 \mathrm{~V}$ 